3 Research Square

\title{
A Novel Optimization Method for Halbach Magnet
}

\author{
Wenjuan Jiang ( $\nabla$ jwjuan2005@163.com ) \\ Northeast Electric Power University \\ Junzhou Li \\ Northeast Electric Power University \\ Didi Sheng \\ Northeast Electric Power University
}

\section{Research Article}

Keywords: Halbach magnet, multilayer, axial adjustment, NMR

Posted Date: August 23rd, 2021

DOl: https://doi.org/10.21203/rs.3.rs-840410/v1

License: (c) (1) This work is licensed under a Creative Commons Attribution 4.0 International License.

Read Full License 


\section{Abstract}

Portable nuclear magnetic resonance (NMR) instruments are widely used in many fields. However, the large volume, low uniformity and end effect limits the application of portable NMR instruments. In order to improve the uniformity and compensate the end effect, a Halbach structure with 9-layer permanent magnet is proposed, which is optimized by axially adjusting the magnet height based on the Halbach array principle and Quality factor $(\mathrm{Q})$ is introduced to represent the magnetic field uniformity at both ends of the central cylinder region. Each layer consists of 16 permanent magnets with trapezoidal cross section and the total volume is $\Phi 240 \times 141.8 \mathrm{~mm}$. Through simulation, it is found that the final magnetic flux density is $1.09 \mathrm{~T}$ and the uniformity is $418 \mathrm{ppm}$ in the central region $(\$ 20 \times 20 \mathrm{~mm})$ of the optimized structure. The proposed structure has the advantages of small size, compactness in structure and homogeneity, which is very suitable for portable NMR systems.

\section{Introduction}

Nuclear magnetic resonance (NMR) technology has been widely used in many fields, such as material science $^{1,2}$, structural biology ${ }^{3}$, medicine $e^{4,5}$ and so on. Magnetic resonance equipment also has the problems of low magnetic field intensity, poor uniformity and low utilization rate of magnetic materials. In order to increase the intensity of the magnetic field required by the NMR equipment, more magnetic materials have to be used in the equipment. This will enlarge the volume and increase the weight of the equipment, which is contradictory to the concept of portable. Since the emergence of new materials such as $\mathrm{NdFeB}$ and $\mathrm{SmCo}^{6}$, it is possible for NMR equipment to be portable.

There are four main structures of portable NMR equipment: single-sided ${ }^{7,8}, \mathrm{C}$-type ${ }^{9}, \mathrm{H}$-type ${ }^{10}$ and Halbach $\operatorname{array}^{11,12}$, in which the Harbach array is use widely due to the characteristics of miniaturization ${ }^{13,14}$. The magnetization directions in the magnetic blocks are different, which makes the magnetic field lines converge on one side and weaken on the other side ${ }^{15,16}$. The magnetic field intensity can be greater than the remanence of permanent magnetic materials and also there is no iron yoke in the structure ${ }^{17,18}$.

Moresi and Magin designed a portable Halbach magnet structure. The magnetic field uniformity is improved by using two high permeability iron plates, but the weight of the whole structure is greatly increased by using iron plates ${ }^{19}$. In 2004, Raich and Blümler proposed a new structure called NMR Mandhala (Magnet Arrangements for Novel Discrete Halbach Layout) by combining theoretical derivation and simulation optimization. The rectangular, circular and polygonal magnets was also suitable to the structure. The proposed NMR Mandhala structure was with mass of $11.4 \mathrm{~kg}$, homogeneity of $700 \mathrm{ppm}$ and center frequency of $12.8 \mathrm{MHz}^{20}$, which produces good uniform using magnetic blocks of the same shape and remanence ${ }^{21}$. However, the volume of the device still greatly limited its application in portable NMR instruments. In 2007, Blümich proposed a method to improve magnetic field homogeneity. They moved the four magnets on the Halbach magnetic ring to $5 \mathrm{~mm}$ along its magnetization direction, which increased the uniformity by 10 times. The magnetic field intensity was $0.22 \mathrm{~T}$ and the homogeneity was 
$0.05 \mathrm{~T} / \mathrm{m}^{22}$. However, this method also reduces the magnetic field intensity. A few years later, B. Blümich et al. placed 8 shimming units to compensate for the end effect ${ }^{23}$. Cooley and Stockmann et al. placed 40 shimming units at both ends of the Halbach structure to optimize the spatial resolution of MRI scanners $^{24}$. In 2011, Windt et al. demonstrated the idea that the Halbach magnetic ring can be opened and closed with the minimum force at a certain angle. The weight of this structure was $3.1 \mathrm{~kg}$, and the magnetic field intensity was $0.57 \mathrm{~T}$. The spherical region with a diameter of $5 \mathrm{~mm}$, in which the uniformity is better than 200 ppm. However, the working area of this structure is too small, which is only suitable for measuring objects such as plants ${ }^{25}$. Later, Menzel and Chen et al. developed a formula, which is used to calculate the magnetic field intensity of equidistant stack ring $26,27$.

In order to design a portable Halbach permanent magnet with good uniformity and effective compensation for end effect. We present an optimization method which can axial adjust the height of the magnet to compensate for the end effect.

The main contribution in this paper are as follows: 1) Only a small amount of magnetic material needs to be added to the original structure to effectively compensate for the end effect and improve uniformity. 2) Quality factor (Q) is introduced to represent magnetic field performance. In ensuring the magnetic field density and uniformity at the same time, through $Q$ as far as possible to play the performance of magnetic materials and reduce volume. 3) The structure is small in size and compact, which is very suitable for portable NMR systems.

The remainder of this paper is organized as follows: First, we explain the principle of Halbach in Section "Halbach array principle". Then, we propose the parameters of the original structure in section "Design". Finally, in section "Simulation method", an novel optimization method with axial adjustment the height of the magnetic block is proposed, which is compared with another optimization method.

\section{Halbach Array Principle}

There are many magnetic blocks in Halbach array which are arranged in order. The magnetic blocks are with different magnetization directions, so the magnetic field lines converges on one side and weakens on the other side ${ }^{15,16}$. As shown in Fig. 1a, theoretical model can be obtained by continuous change of magnetization direction according to specific rules. In ideal case, a transverse magnetic field can be generated in the central region of the structure and the magnetic fields at each point are equal. Its magnetic field intensity ${ }^{16} B_{0}$ is given by Eq. (1):

$$
B_{0}=B_{r} \ln \left(r_{\text {out }} / r_{\text {in }}\right)
$$

Where, $B_{r}$ is the remanence of magnetic material, $r_{\text {out }}$ and $r_{\text {in }}$ are the outer radius and inner radius of the Halbach array, respectively. 
Due to the actual machining problems and the magnetization direction of the magnetic block, it is difficult to have a continuously changing magnetization direction in the magnetic ring. Therefore, in practice, several magnetic blocks are spliced together to construct a discrete structure to approximately replace the theoretical model ${ }^{28}$. As shown in Fig. $1 \mathrm{~b}$, the magnetization direction angle $\beta$ of magnetic block in discrete structure varies twice as much as the rotation angle $\theta$. The magnetization angle can be given by Eq. (2):

$$
\beta_{i}=2 \theta_{i}
$$

where, $i=0,1, \ldots, M-1, \theta=2 \pi / M$, and $M=2^{P}, P$ is an integer greater than $2 . M$ is the number of permanent magnet blocks contained in a magnetic ring.

Figure $1 \mathrm{~b}$ is a Halbach array formed by 16 sector magnetic blocks. The central magnetic field intensity $B_{0}$ of discrete structure is given by Eq. (3):

$$
B_{0}=B_{r} \frac{\sin (2 \pi / M)}{2 \pi / M} \ln \left(r_{\text {out }} / r_{\text {in }}\right)
$$

It can be observed from Eq. (3) that the larger $M$ is, the closer Eq. (3) is to Eq. (1) ${ }^{29}$. So, $M$ is chosen 16 in this paper.

In Fig. 1 it is found that the magnetic block with trapezoidal cross section is closer to the theoretical model, and the trapezoidal cross section is convenient for machining. Therefore, the magnetic block with trapezoidal cross section is selected to construct the Halbach array in this paper. The partial structure of the magnetic ring is shown in Fig. 2, and the height of the trapezoidal section $d=r_{\text {out }}-r_{i n}$, the width of the baseline $a$ and the topline $b$ are $a=r_{\text {in }} \tan (\theta / 2)$ and $b=r_{\text {out }} \tan (\theta / 2)$, respectively ${ }^{30}$.

If your manuscript reports research with non-human animals, the Methods section starts with a statement confirming that the research complies with all relevant ethical regulations; naming the board and institution that approved the study protocol; and confirming that the ARRIVE guidelines were used to report the research.

\section{Design}

The purpose of this study is to generate magnetic field intensity greater than $1 \mathrm{~T}$ in the central region to satisfy the detection requirements. The structural height is required to be $150 \mathrm{~mm}$, and the uniformity in the central region $(\Phi 20 \times 20 \mathrm{~mm})$ is required to be $500 \mathrm{ppm}$.

A permanent magnet structure with $r_{\text {in }}=30 \mathrm{~mm}$ and $r_{\text {out }}=120 \mathrm{~mm}$ was designed in previous work. The magnet material was NdFeB-48M, with coercivity $H_{c}=1027 \mathrm{kA} / \mathrm{m}$ and remanence $B_{r}=1.38 \mathrm{~T}$. In the 
research work of Soltner and Blisümler ${ }^{24}$, it is known that single-layer Halbach magnet structure cannot construct a relatively uniform magnetic field in the central region due to its limited height. Therefore, in order to improve the uniformity, 9-layers of Halbach magnetic rings are considered. Each layer consists of 16 trapezoidal magnetic blocks. The height of the magnetic ring is $10 \mathrm{~mm}$ and the interval between two magnetic rings is $6 \mathrm{~mm}$. The final structure has a size of $\Phi 240 \times 138 \mathrm{~mm}$. The permanent magnet structure was constructed in Ansys Workbench and Ansys Maxwell. The simulation results are that the maximum magnetic field intensity is $1.027 \mathrm{~T}$ and the homogeneity is $8612 \mathrm{ppm}$.

When the magnet is short in height, which will cause the end effect, resulting in poor uniformity. The magnetic field intensity at both ends of the central region is small, as shown in Fig. 3a. The magnetic field intensity in the central part of the region is large, as shown in Fig. 3b. The key to compensating for the end effect is to improve the uniformity at both ends of the region. The uniformity of the two ends of the central region is marked $H_{1}$, and $H_{1}$ is given by Eq. (4):

$$
H_{1}=\frac{B_{\max }-B_{\min }}{B_{\text {avenge }}} \times 10^{6}
$$

Where, $B_{\max }$ and $B_{\min }$ represent the maximum and minimum magnetic field intensity at both ends of the central region, respectively. $B_{\text {average }}$ represents the average value of the magnetic field intensity at both ends of the central region. Through calculation, the $H_{1}$ of the structure designed in the previous section is $3733 \mathrm{ppm}$.

\section{Simulation Method}

In order to compensate the end effect, an optimization method is proposed in section "Simulation method". The optimization method we propose is divided into two parts: the part 1 is to raise the height of the outer layer and the part 2 is to reduce the height of the inner layer.

Before optimizing, we named the magnetic rings and the magnetic blocks. As shown in Fig. 4a, the 1-3 layers and the 7-9 layers are named the outer layer, and the 4-6 layers are named the inner layer. Then we name the first and ninth layers as Level 1. Accordingly, we named the 2nd and 8th layers as Level 2, and so on until Level 5. Each level is symmetrical about Level 5. Each layer of magnetic ring has 16 magnetic blocks. There are 8-pairs of magnetic blocks with the same magnetization direction. These 8-pairs of magnetic blocks are shown in Fig. 4b.

During optimization, the height of each magnetic block should be adjusted axially, and the uniformity change of the central cylinder area $(\Phi 20 \times 20 \mathrm{~mm})$ should be analyzed. Finally adjust the height of magnetic block according to a quality factor (Q), and Q given by Eq. (5): 


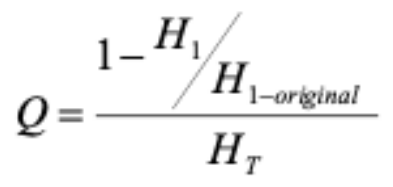

Where, $H_{1}$ represents the uniformity at both ends of the region. $H_{1-\text { original }}$ represents the uniformity of both ends of the region before this level of optimization. The molecule represents the ability to improve the magnetic field uniformity at both ends after the height of the magnetic block changing. $H_{T}$ represents the sum of the height of these magnets. Q represents the ability of unit magnetic block to improve the uniformity at both ends of the region. In ensuring the magnetic field density and uniformity at the same time, through $\mathrm{Q}$ as far as possible to play the performance of magnetic materials and reduce volume.

\section{Simulation Results}

For the optimization of the part 1, we axially adjust the height of Level 1. Each time the height of a pair of magnets is adjusted from $10.1 \mathrm{~mm}$ to $13.9 \mathrm{~mm}$, and the interval is $0.1 \mathrm{~mm}$. As shown in Table 1, the second column represents the best uniformity of the magnetic block at this height. The specific value of uniformity is represented by the third column. The fourth column represents the $\mathrm{Q}$ of the magnetic block at this height, where the $\mathrm{Q}$ is maximum. The last column represents the sum of quality factors for the period from $10.1 \mathrm{~mm}$ to $13.9 \mathrm{~mm}$ in height, which is called $Q_{\text {Total }}$.

As the first group of experiments, we selected the largest pair 5 and pair 1 in $Q_{T o t a l}$ and adjusted their height from $10.1 \mathrm{~mm}$ to $14 \mathrm{~mm}$ at the same time. The remaining magnetic block height is set to $10 \mathrm{~mm}$. As the second group of experiments, we selected pair 5, pair 1 and pair 8 , which are the top three pairs of magnetic blocks in $Q_{\text {Total, }}$ and adjusted their heights from $10.1 \mathrm{~mm}$ to $14 \mathrm{~mm}$ at the same time. The remaining magnetic block height is set to $10 \mathrm{~mm}$. Accordingly, there are seven groups of experiments. Seven groups of experiments are selected as shown in Table 2.

From the fifth column of Table $2, Q$ is gradually decreasing. Only the $Q$ of the fourth group of experiments is increased compared with that of the previous group. For the two ends of the structure, increasing the amount of magnetic materials at both ends of the magnetic ring will improve the uniformity of the central region. So we want to add as much the amount of magnetic material as possible to both ends of the magnetic ring. And through the quality factor as far as possible to play the

performance of magnetic materials and reduce volume. So we choose the magnetic block height represented by the fourth group of experiments as the magnetic block height of Level 1 . The height of the five pairs of magnets is determined to be $13.8 \mathrm{~mm}$. The remaining magnetic blocks height are set to 10 $\mathrm{mm}$. The optimized results are that the $H_{1}$ of Level 1 is $2730 \mathrm{ppm}$ and the uniformity of this structure is $6711 \mathrm{ppm}$.

This optimization method is used to Level 2 to Level 5 to improve uniformity. The adjustment is shown in Table 3. It can be seen from Table 3 that the magnetic field uniformity is effectively improved by axial 
adjustment.

It can be found from Figs. 5a,b that the original structure and the optimized structure have little difference in the magnetic field intensity, but the uniformity of the original structure is poor. The magnetic field distribution of the optimized structure is shown in Figs. 6a,b. The optimized structure uses $13.38 \%$ more magnetic materials than the original structure, and the uniformity is improved by $95.17 \%$. The maximum magnetic field intensity is $1.09 \mathrm{~T}$ and the homogeneity is $418 \mathrm{ppm}$. The whole structure has a size of $\Phi 240 \times 141.8 \mathrm{~mm}$.

In order to show the correctness of the optimization method, we set up a compare group. The optimization process of the compare group is to adjust the magnetic block height of each magnetic ring. All magnetic blocks in magnetic ring are of the same height. The height of the magnetic ring was adjusted from $10.1 \mathrm{~mm}$ to $14 \mathrm{~mm}$ with an interval of $0.1 \mathrm{~mm}$. The results are shown in Table 4. The compare group used more $18.89 \%$ magnetic materials than the original structure, and the uniformity was increased by $95.84 \%$. The compare group used more magnetic materials than optimized structure. But their magnetic field intensity and uniformity have little difference. Through simulation, it is found that if the magnetic block in the magnetic ring is at the same height, the performance of magnetic materials cannot be maximized. The proposed optimization method can ensure the magnetic field density and uniformity at the same time, as far as possible to play the performance of magnetic materials.

\section{Discussion}

We present an optimization method which can axial adjust the height of the magnet to compensate for the end effect. Only a small amount of magnetic material needs to be added to the original structure to effectively compensate for the end effect and improve uniformity. In ensuring the uniformity at the same time, through the quality factor (Q) as far as possible to play the performance of magnetic materials and reduce volume. The optimized structure uses $13.38 \%$ more magnetic materials than the original structure, and the uniformity is improved by $95.17 \%$. The optimized permanent magnet structure generated a $1.09 \mathrm{~T}$ magnetic field intensity and the uniformity is $418 \mathrm{ppm}$. Moreover, this method can be combined with genetic algorithm to more accurately adjust the height of the magnetic block and improve uniformity. Then we will build the halbach multilayer permanent magnet structure based on the optimization model, and further test its performance.

\section{Data availability}

All data generated or analyzed during this study are included in this published article.

\section{Declarations}

Acknowledgements 
This work is supported by Science and Technology Project of "the 13th five-year plan" of Jilin Province Education Department: JJKH20200120KJ.

\section{Author contributions}

The calculations and simulations were performed by J. L., and the manuscript was authored by J. L. and D. S. The research was directed by W. J.

\section{Competing interests}

The authors declare no competing interests.

\section{Additional information}

Correspondence and requests for materials should be addressed to W. J.

Reprints and permissions information is available at www.nature.com/reprints.

Publisher's note Springer Nature remains neutral with regard to jurisdictional claims in published maps and institutional affiliations.

\section{References}

1. Fleury, M., Berthe, G. \& Chevalier, T. Diffusion of water in industrial cement and concrete sciencedirect. Magn. Reson. Imaging. 56, 32-36 (2019).

2. Adams, A. Non - destructive analysis of polymers and polymer - based materials by compact nmr. Magn. Reson. Imaging. 56, 119-125 (2019).

3. Franks, W. T., Linden, A. H., Kunert, B., Rossum, B. \& Oschkinat, H. Solid - state magic - angle spinning nmr of membrane proteins and protein - ligand interactions. Eur. J. Cell. Biol. 91(4), 340348 (2012).

4. Zalesskiy, S. S., Danieli, E., Blumich, B. \& Ananikov, V. P. Miniaturization of nmr systems: Desktop spectrometers, microcoil spectroscopy, and "nmr on a chip" for chemistry, biochemistry, and industry. Chem. Rev. 114(11), 5641-5694 (2014).

5. Luo, Y. \& Alocilja, E. C. Portable nuclear magnetic resonance biosensor and assay for a highly sensitive and rapid detection of foodborne bacteria in complex matrices. J. Biol. Eng. 11(1), 1-8 (2017).

6. Sagawa, M., Fujimura, S., Yamamoto, H., Matsuura, Y. \& Hiraga, K. Permanent magnet materials based on the rare earth-iron-boron tetragonal compounds. IEEE. T. Magn. 20(5), 1584-1589 (1984).

7. Bashyam, A., Li, M. \& Cima, M. J. Design and experimental validation of unilateral linear halbach magnet arrays for single - sided magnetic resonance. J. Magn. Reson. 292, 36-43 (2018).

8. Mcdaniel, P. C., Cooley, C. Z., Stockmann, J. P. \& Wald, L. L. The mr cap: A single - sided mri system designed for potential point - of - care limited field - of - view brain imaging. Magn. Reson. Med. 
82, 1946-1960 (2019).

9. Überrück, T. \& Blümich, B. Variable magnet arrays to passively shim compact permanent - yoke magnets. J. Magn. Reson. 298, 77-84 (2019).

10. Alnajjar, B. M., Buchau, A., Anders, J. \& Blümich, B. An h - shaped low - field magnet for nmr spectroscopy designed using the finite element method. Int. J. Appl. Electrom. 60(S1), S3-S14 (2019).

11. Cooley, C. Z. et al. Design of sparse halbach magnet arrays for portable mri using a genetic algorithm. IEEE. T. Magn. 54(1), 1-12 (2017).

12. Yu, P. et al. A low - cost home-built nmr using halbach magnet. J. Magn. Reson. 294, 162-168 (2018).

13. Danieli, E., Perlo, J., Blümich, B. \& Casanova, F. Small magnets for portable nmr spectrometers. Angew. Chem. Int. Ed. 49(24), 4133-4135 (2010).

14. Lei, M. Z., Dai, W. Z. \& Wang, L. Q. Analysis on a novel halbach-type transverse-flux linear oscillatory motor for linear compressor. Appl. Mech. Mater. 241-244, 1431-1437 (2003).

15. Halbach, K. Strong rare earth cobalt quadrupoles. IEEE. T. Nucl. Sci. 26(3), 3882-3884 (1979).

16. Halbach, K. Design of permanent multipole magnets with oriented rare earth cobalt material. Nucl. Instrum. Methods. 169(1), 1-10 (1980).

17. Kumada, M. et al. Development of 4 tesla permanent magnet. In PACS2001. Proceedings of the 2001 Particle Accelerator Conference (Cat. No. 01CH37268), vol. 5, 3221-3223 (IEEE, 2001).

18. Lu, R., Yi, H., Wu, W., Jiang, Y. \& Ni, Z. Optimization and experimental test of a miniature permanent magnet structure for a microfluidic magnetic resonance chip. Int. J. Appl. Electrom. 42(4), 479-489 (2013).

19. Moresi, G. \& Magin, R. Miniature permanent magnet for table - top nmr. Concepts Magn. Reson. Part B: Magn. Reson. Eng. An Educ. J. 19(1), 35-43 (2003).

20. Raich, H. \& Blümler, P. Design and construction of a dipolar halbach array with a homogeneous field from identical bar magnets: Nmr mandhalas. Concepts Magn. Reson. Part B: Magn. Reson. Eng. An Educ. J. 23(1), 16-25 (2004).

21. Soltner, H. \& Blümler, P. Dipolar halbach magnet stacks made from identically shaped permanent magnets for magnetic resonance. Concept. Magn. Reson. A. 36(4), 211-222 (2010).

22. Anferova, S. et al. Improved halbach sensor for nmr scanning of drill cores. Magn. Reson. Imaging. 25(4), 474-480 (2007).

23. Danieli, E., Mauler, J., Perlo, J., Blümich, B. \& Casanova, F. Mobile sensor for high resolution nmr spectroscopy and imaging. J. Magn. Reson. 198(1), 80-87 (2009).

24. Cooley, C. Z. et al. Two - dimensional imaging in a lightweight portable mri scanner without gradient coils. Magn. Reson. Med. 73(2), 872-883 (2015).

25. Windt, C. W., Soltner, H., Van Dusschoten, D. \& Blümler, P. A portable halbach magnet that can be opened and closed without force: the nmr - cuff. J. Magn. Reson. 208(1), 27-33 (2011). 
26. Menzel, K., Windt, C. W., Lindner, J. A., Michel, A. \& Nirschl, H. Dipolar openable halbach magnet design for high - gradient magnetic filtration. Sep. Purif. Technol. 105, 114-120 (2013).

27. Chen, Q., Zhang, G., Xu, Y. \& Yang, X. Design and simulation of a multilayer halbach magnet for nmr. Concepts Magn. Reson. Part B: Magn. Reson. Eng. 45(3), 134-141 (2015).

28. Coey, J. M. D. Permanent magnet applications. J. Magn. Magn. Mater. 248(3), 441-456 (2002).

29. Turek, K. \& Liszkowski, P. Magnetic field homogeneity perturbations in finite halbach dipole magnets. J. Magn. Reson. 238, 52-62 (2014).

30. Hu, J. et al. Design of a multilayer halbach permanent magnet for human finger nmr detection. Int. J. Appl. Electromagn. Mech. 54(3), 315-327 (2017).

\section{Tables}

\begin{tabular}{|lllll|}
\hline Pair & Height $(\mathrm{mm})$ & $H_{1}(\mathrm{ppm})$ & $\mathrm{Q}$ & $Q_{\text {Total }}$ \\
\hline 1 & 13.8 & 3473 & $0.126 \%$ & $1.58 \%$ \\
\hline 2 & 13.6 & 3515 & $0.107 \%$ & $0.94 \%$ \\
\hline 3 & 13.9 & 3557 & $0.085 \%$ & $0.56 \%$ \\
\hline 4 & 13.4 & 3480 & $0.126 \%$ & $1.16 \%$ \\
\hline 5 & 13.8 & 3436 & $0.144 \%$ & $1.86 \%$ \\
\hline 6 & 13.4 & 3520 & $0.107 \%$ & $1.31 \%$ \\
\hline 7 & 13.8 & 3530 & $0.099 \%$ & $0.43 \%$ \\
\hline
\end{tabular}

Table 1. Compare the magnetic performance of different pairs at different heights. 


\begin{tabular}{|llllll|}
\hline $\begin{array}{l}\text { Experiment } \\
\text { group }\end{array}$ & Pair & $\begin{array}{l}\text { Height } \\
(\mathrm{mm})\end{array}$ & $\begin{array}{l}H_{1} \\
(\mathrm{ppm})\end{array}$ & $\mathrm{Q}$ & $\begin{array}{l}\text { Uniformity of the central } \\
\text { region }(\mathrm{ppm})\end{array}$ \\
\hline 1 & pair 5,1 & 13.9 & 3199 & $0.128 \%$ & 7705 \\
\hline 2 & pair $5,1,8$ & 13.8 & 3050 & $0.111 \%$ & 7343 \\
\hline 3 & pair $5,1,8,6$ & 13.8 & 2979 & $0.091 \%$ & 7163 \\
\hline 4 & pair $5,1,8,6,4$ & 13.8 & 2730 & $0.097 \%$ & 6711 \\
\hline 5 & pair $5,1,8,6,4,2$ & 13.9 & 2588 & $0.091 \%$ & 6396 \\
\hline 6 & pair & 13.9 & 2414 & $0.090 \%$ & 5879 \\
\hline 7 & $5,1,8,6,4,2,3$ & & & & \\
\hline
\end{tabular}

Table 2. Compare the magnetic performance of different pairs at different heights.

\begin{tabular}{|lllll|}
\hline Level & Pair & Height $(\mathrm{mm})$ & $H 1(\mathrm{ppm})$ & Uniformity of the central region $(\mathrm{ppm})$ \\
\hline 2 & pair $5,1,6,4,8,2,3,7$ & 13.8 & 403 & 1639 \\
\hline 3 & pair $6,5,2,8$ & 10.3 & 398 & 1419 \\
\hline 5 & pair $6,5,8,7,2,4,3$ & 9.6 & 206 & 487 \\
\hline 4 & pair 6 & 9.9 & 145 & 418 \\
\hline
\end{tabular}

Table 3. Magnetic properties after axial adjustment of each level.

\begin{tabular}{|llll|}
\hline Level & Height $(\mathrm{mm})$ & $H_{1}(\mathrm{ppm})$ & Uniformity of the central region $(\mathrm{ppm})$ \\
\hline 1 & 14 & 2368 & 5684 \\
2 & 14 & 192 & 526 \\
3 & 10.2 & 302 & 462 \\
4 & 10.3 & 133 & 358 \\
\hline
\end{tabular}

Table 4. Magnetic properties after axial adjustment of each level.

Figures 

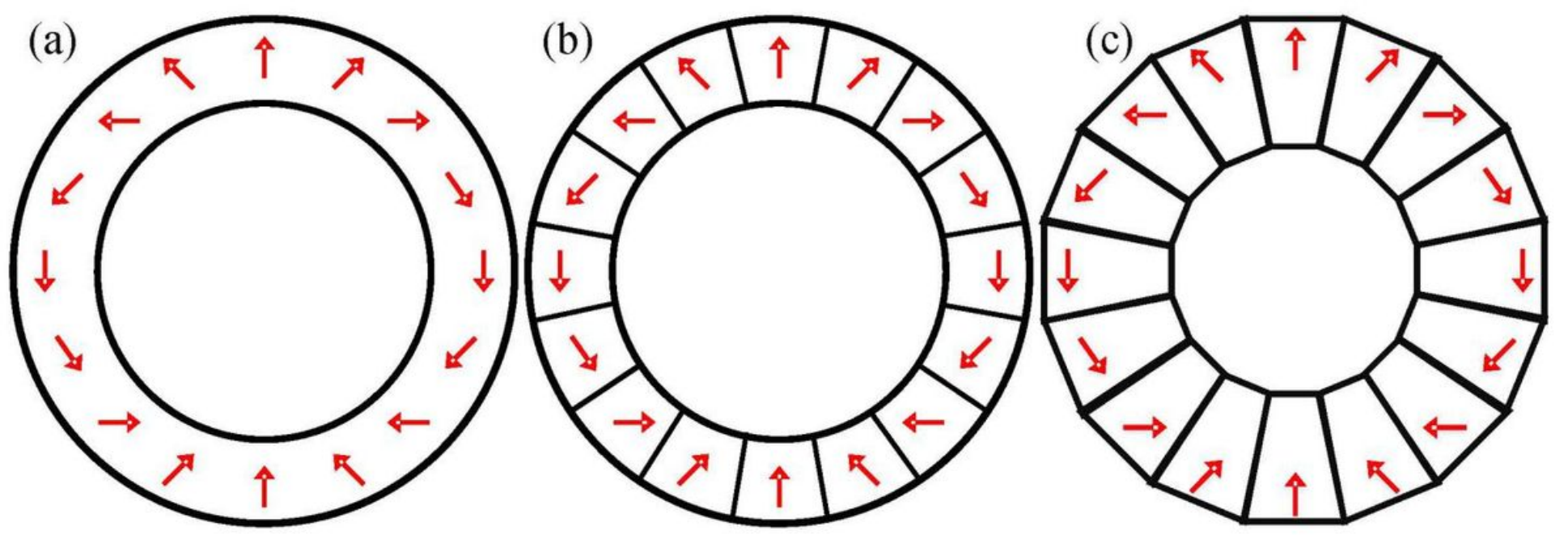

Figure 1

(a) Theoretical model; (b) Discrete structure of theoretical model; (c) Trapezoid section. 


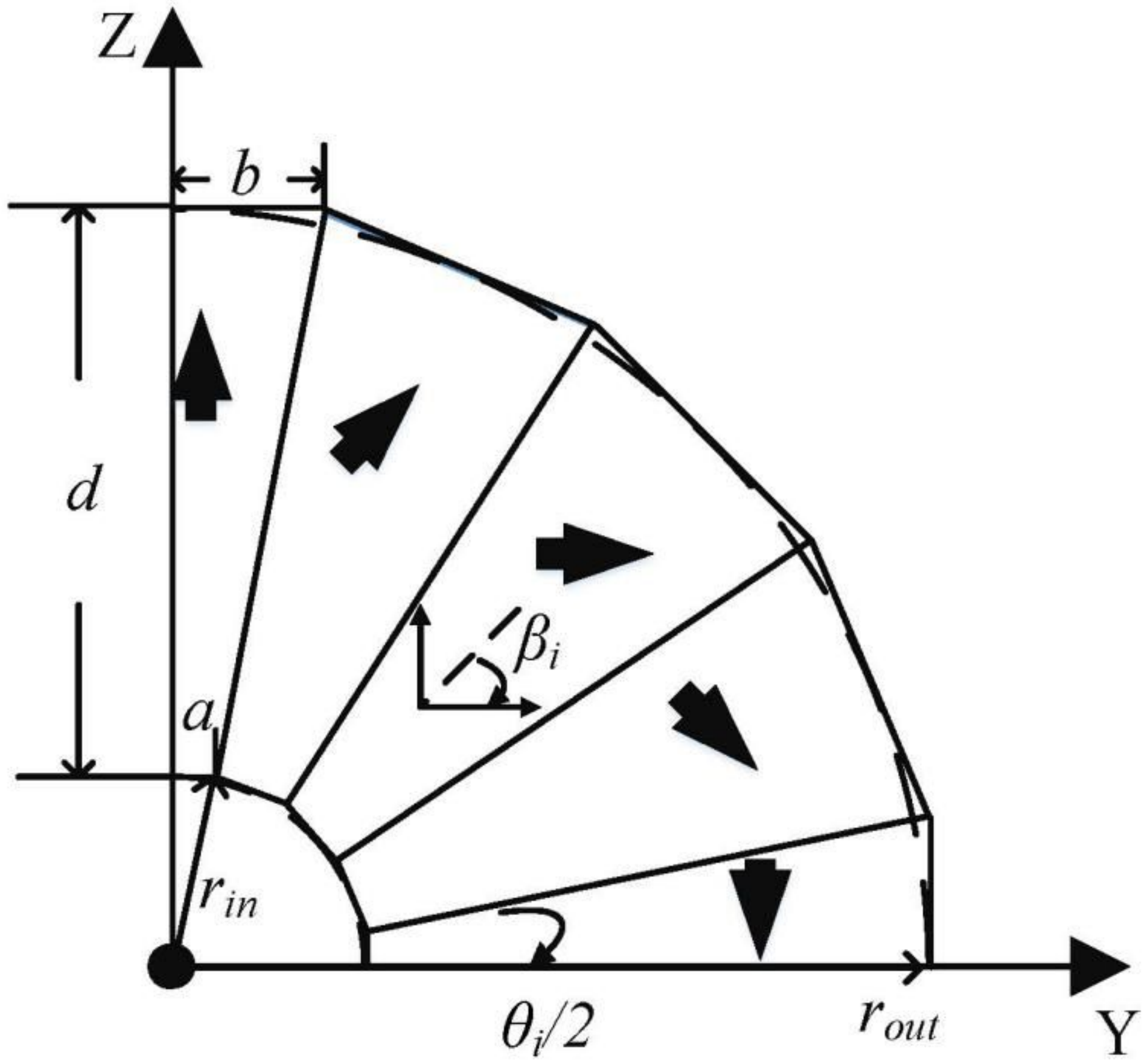

Figure 2

Halbach array of trapezoidal magnetic blocks. 
(a)

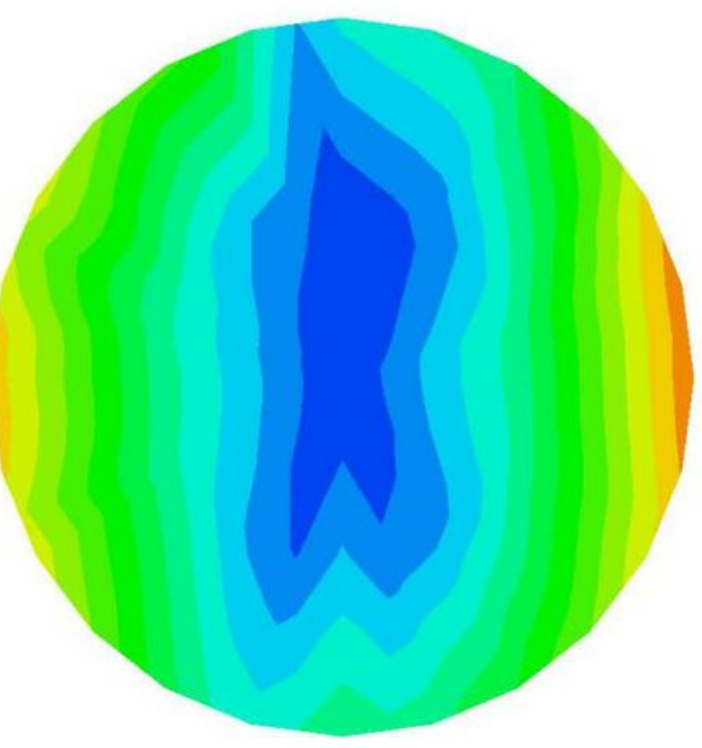

(b)

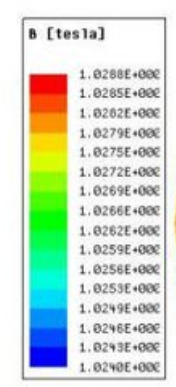

\section{Figure 3}

(a) The magnetic field distribution at both ends of the central region; (b) Magnetic field distribution on central section of central region.

(a)

Layer number

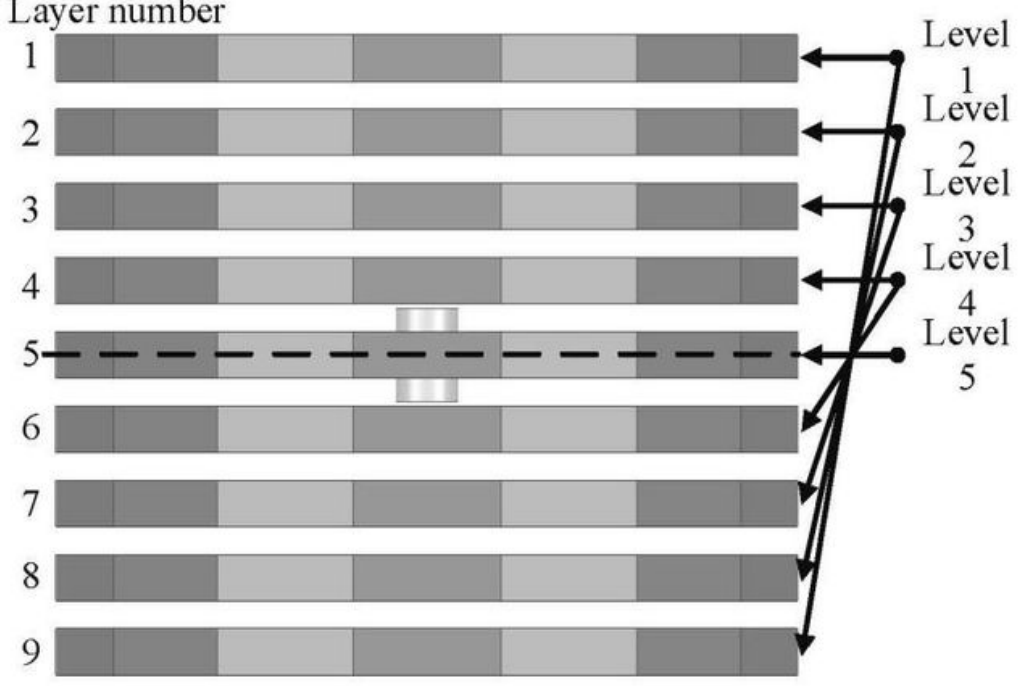

(b)

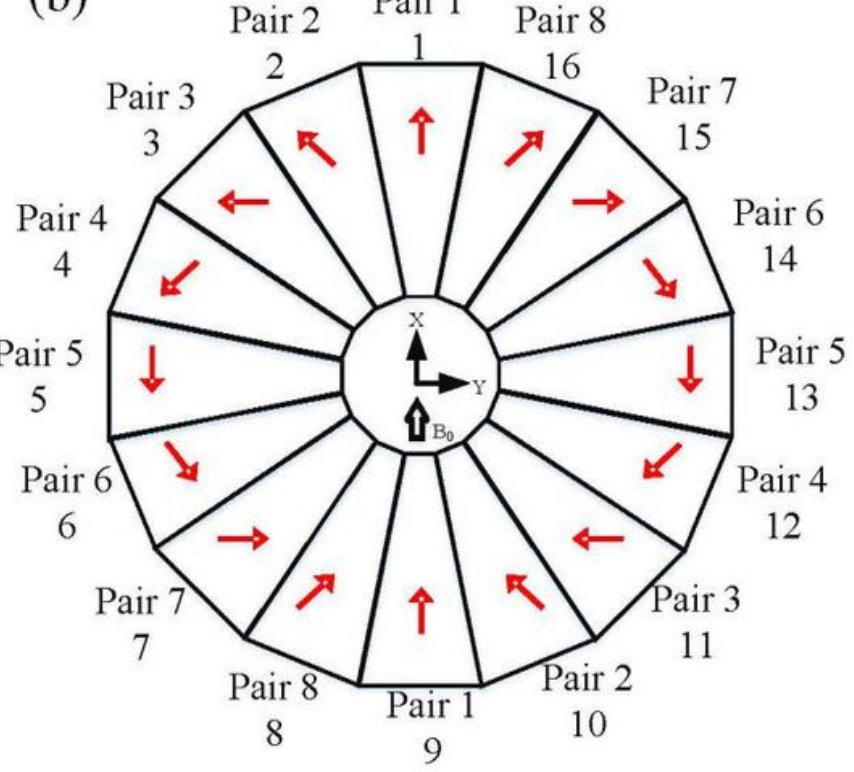

\section{Figure 4}

(a) Schematic representation of the magic ring; (b) Schematic representation of the magnet blocks. 

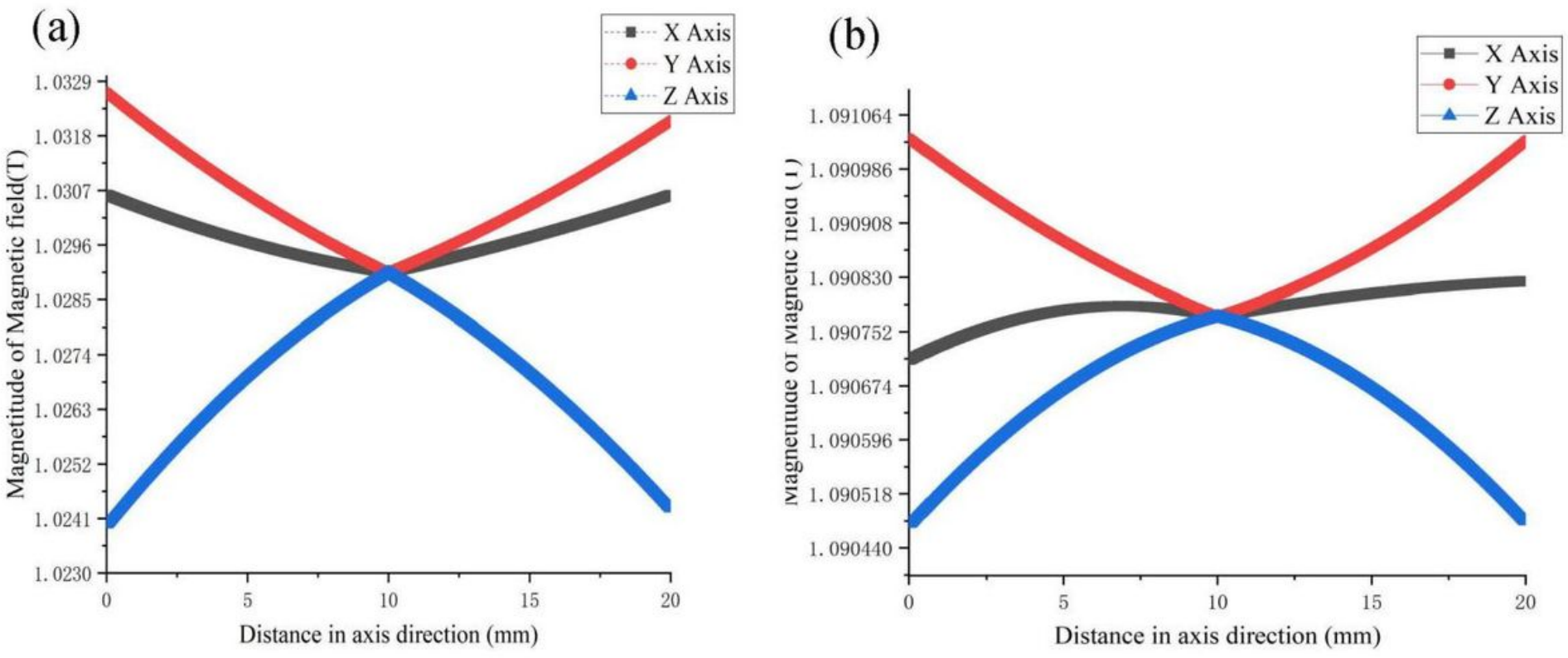

\section{Figure 5}

(a) The magnetic field intensity of the original results changing along the coordinate axis directions; (b) The magnetic field intensity of the optimization results changing along the coordinate axis directions.
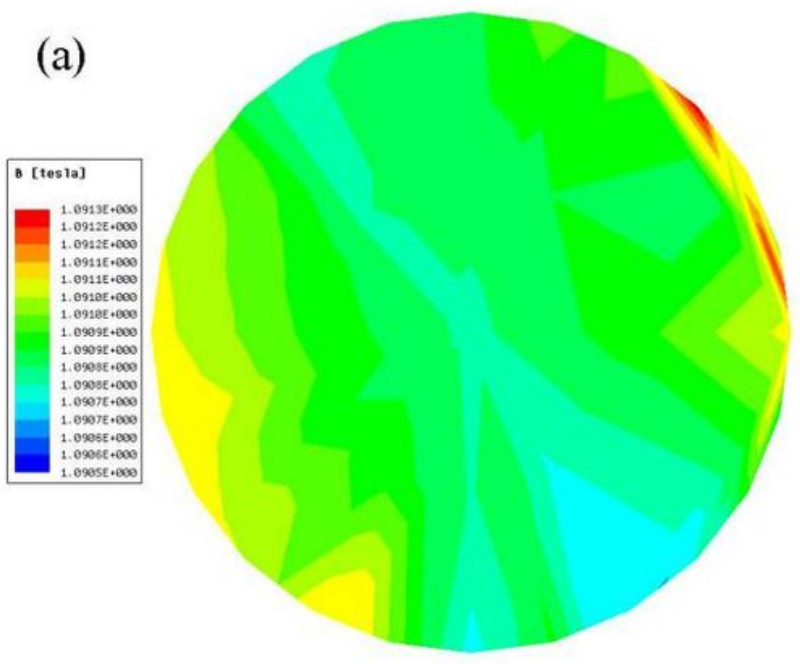

(b)

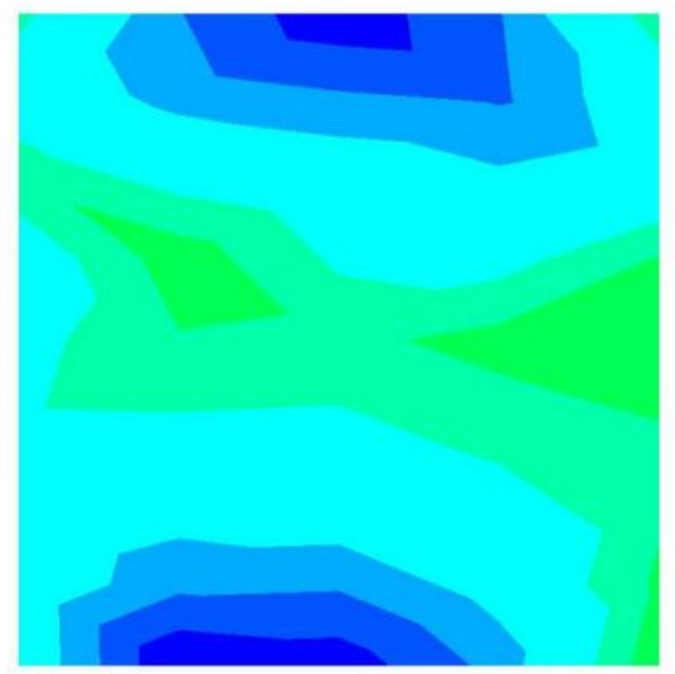

Figure 6

(a) The magnetic field distribution on xy plane of the central region; (b) The magnetic field distribution on $x z$ plane of the central region. 